\title{
A modular, self-assembling metallaphotocatalyst for cross couplings using the full visible-light spectrum
}

\author{
Authors: Susanne Reischauer, ${ }^{1,2}$ Volker Strauss, ${ }^{3}$ Bartholomäus Pieber ${ }^{1 *}$
}

\author{
Affiliations: \\ ${ }^{1}$ Department of Biomolecular Systems, Max Planck Institute of Colloids and Interfaces, Am \\ Mühlenberg 1, 14476 Potsdam, Germany \\ ${ }^{2}$ Department of Chemistry and Biochemistry, Freie Universität Berlin, Arnimalle 22, 14195 \\ Berlin, Germany \\ ${ }^{3}$ Department of Colloid Chemistry, Max Planck Institute of Colloids and Interfaces, Am \\ Mühlenberg 1, 14476 Potsdam, Germany \\ *Correspondence to: bartholomaeus.pieber@mpikg.mpg.de
}

\begin{abstract}
The combination of nickel- and photocatalysis has unlocked a variety of cross couplings. These protocols rely on a few photocatalysts that can only convert a small portion of visible light $(<500 \mathrm{~nm})$ into chemical energy. Many dyes that absorb a much broader spectrum of light are not applicable due to their short-lived excited states. Here we describe a self-assembling catalyst system that overcomes this limitation. The modular approach combines nickel catalysis with dye-sensitized titanium dioxide and can be used to catalyze various bond formations.
\end{abstract}


The combination of photo- and nickel catalysis (metallaphotocatalysis) has emerged as a powerful strategy for carbon-carbon and carbon-heteroatom cross couplings (Fig. 1A) (1-3). Key to the success are redox or photosensitization events between a nickel- and a photocatalyst (PC). Applicable PCs are iridium and ruthenium polypyridyl complexes (2), or carbazolyl dicyanobenzenes (4), with tailored redox potentials or triplet energies, and long-lived excited states (Fig. 1B). These PCs are limited to short excitation wavelengths that can cause unwanted side-reactions (5). These could be avoided in a metallaphotocatalytic system that harvests longer wavelengths. Current approaches towards photocatalysis with low photon energies require complex catalytic cocktails that enable photon upconversion $(6)$, osmium complexes as PCs (7), or multi-photon excitation processes (5).

The use of abundant dyes that absorb broadly across the visible-light spectrum is highly desirable. The redox potentials and triplet energies of many commodity chemicals, such as fluorescein, rose bengal, or coumarins are in theory suitable for metallaphotocatalysis (8), but their short excited state lifetime render a diffusion-limited interaction with a nickel catalyst in a homogeneous solution unlikely (Fig. 1B). These dyes are, however, able to sensitize metal oxide semiconductors, such as $\mathrm{TiO}_{2}$, in dye-sensitized solar cells (DSSCs) (9), or dye-sensitized photocatalysts (DSPs) for light-driven $\mathrm{H}_{2}$ production (10). The carboxylic acid groups of the dyes bind to the surface hydroxyl groups of $\mathrm{TiO}_{2}$. This facilitates electron injection into the conduction band of the semiconductor upon photoexcitation. This results in a charge-separated species that is sustained for several microseconds (11).

We wondered whether immobilization of a suitable nickel complex on dye-sensitized $\mathrm{TiO}_{2}$ accesses a bifunctional material that serves as metallaphotocatalyst (Fig. $1 \mathrm{C}$ ). We hypothesized that such a system overcomes limitations related to short excited state lifetimes and diffusion-controlled energy or single electron transfer events due to the close spatial proximity between the PC and the nickel catalyst. The proposed, modular design of dyesensitized metallaphotocatalysts (DSMPs) allows selecting dyes/wavelengths and nickel complexes depending on the respective application. 

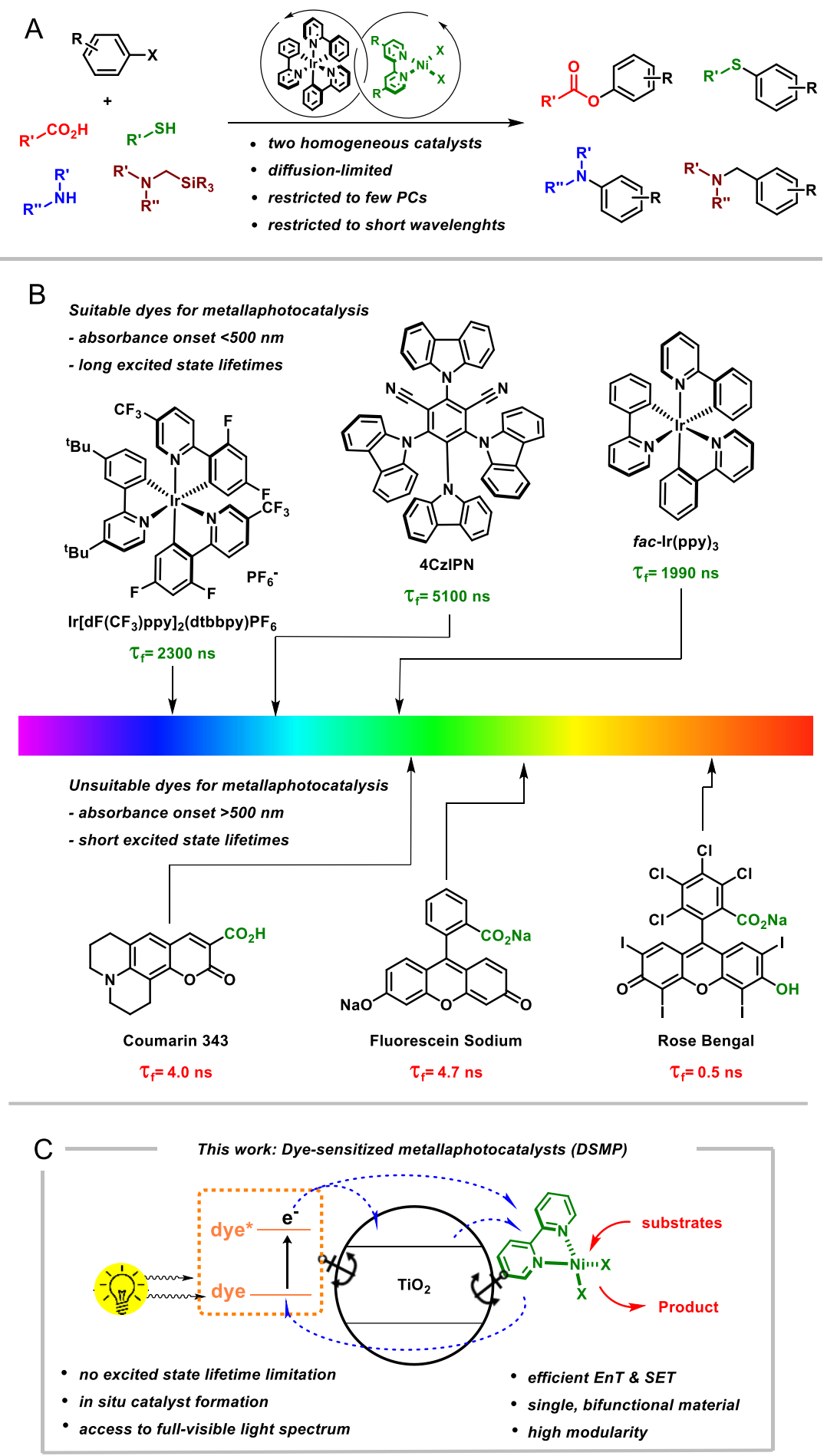

Fig. 1. Working hypothesis towards a modular, heterogeneous metallaphotocatalyst. Cross-coupling reactions using homogeneous metallaphotocatalysis (A). Onset of absorption of photocatalysts. The suitability for homogeneous metallaphotocatalysis depends on excited state lifetime (B). Dye-sensitized metallaphotocatalysts (DSMP) are proposed to overcome excited state lifetime limitations $(\mathbf{C})$. 
Our investigations started with the $O$-arylation of carboxylic acids that was reported using the PC $\operatorname{Ir}(\mathrm{ppy})_{3}$ (ppy = 2-phenylpyridine) and a nickel bipyridine complex. The crosscoupling proceeds via an energy transfer (EnT) mechanism (12), and is feasible using semiconductors that absorb blue light $(13,14)$, but does not work using simple organic dyes (4). We first tested if anchoring of a nickel complex on $\mathrm{TiO}_{2} \mathrm{P} 25$ results i) in an active, heterogeneous metallaphotocatalyst, and ii) improves the reaction yield due to permanent spatial proximity of the PC and the nickel catalyst using near-UV light. A ligand equipped with carboxylic acid groups (dcbpy = 2,2'-bipyridine-4,4'-dicarboxylic acid) indeed gave a higher yield of the $\mathrm{C}-\mathrm{O}$ cross coupling product $\mathbf{1}$ than a ligand that lacks functionalities capable of binding to the semiconductor's surface (dtbbpy $=4,4^{\prime}$-di-tert-butyl-2,2'-dipyridyl) (Fig. 2A). Next, dyes that contain a suitable anchoring group were studied as sensitizers using green light $(525 \mathrm{~nm})$. Fluorescein sodium (NaFluo) showed the best results (see Supplementary Information). The $\mathrm{C}-\mathrm{O}$ coupling was also feasible at higher wavelengths (666 nm) using the ruthenium dye N3 that has an excited state lifetime of $20 \mathrm{~ns}$ (9). The DSMPs self-assemble in situ. An ex situ preparation of the DSMPs was carried out to characterize the bifunctional materials (Fig. 2B). The UV-Vis spectra of the materials confirmed immobilization of the dyes on the metal oxide, and inductively coupled plasma - optical emission spectrometry (ICP-OES) corroborated anchoring of the nickel complex.

Optimization of all reaction parameters using the in situ DSMP approach resulted in a highly selective formation of 1 using blue $(440 \mathrm{~nm})$, green $(525 \mathrm{~nm})$, or red $(666 \mathrm{~nm})$ light (Fig. 2C). The highest activities were obtained when the amount of dye $(1.25 \mathrm{~mol} \%)$ and $\mathrm{NiCl}_{2} \cdot \mathrm{dcbpy}$ (10 mol\%) exceeded the loading that was determined using ex situ DSMP preparation. This is rationalized by a dynamic equilibrium between immobilized and unbound $\mathrm{NiCl}_{2} \cdot \mathrm{dcbpy}$ as well as dye molecules, which also contribute to productive catalysis in the course of the bulk experiment (10). No product formation was observed in the absence $\mathrm{TiO}_{2} \mathrm{P} 25$, the dye, dcbpy, nickel salt, or light.

The DSMP approach also enabled metallaphotocatalyzed C-S (15), C-N (16), and CC (17) bond formations that proceed via single electron transfer processes (SET) (Fig. 2D). The modular design principle and the self-assembling strategy facilitated a straightforward optimization of dyes, nickel salts, and ligands resulting in selective cross-couplings using blue $(440 \mathrm{~nm})$, green $(525 \mathrm{~nm})$, and red $(666 \mathrm{~nm})$ light. With regards to the $\mathrm{C}-\mathrm{C}$ coupling, $68 \%$ of the desired product (4) were obtained using blue light, which is similar to the homogeneous metallaphotocatalysis system (17). Reactions at higher wavelengths, not accessible with the original, homogeneous approach, resulted in a higher selectivity. 
A

Amproved yield through surface binding

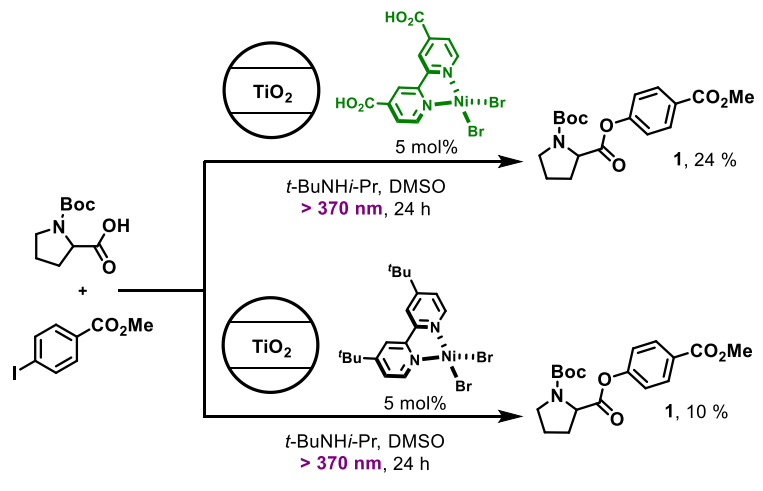

C Optimized conditions and control studies for C-O cross-coupling
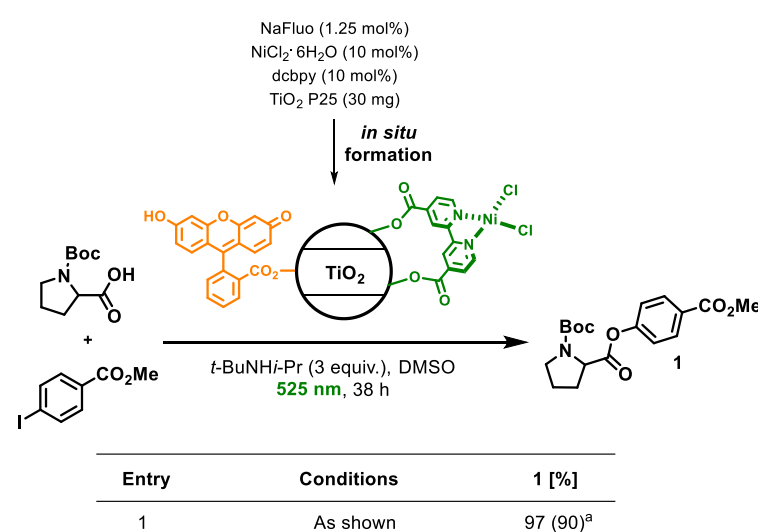

$2 \quad$ Fluo-DSMP prepared ex situ 63

$3 \quad \mathrm{NoTiO}_{2}$ n.d

$4 \quad$ No dye n.d.

$5 \quad$ No dcbpy trace

$6 \quad \mathrm{No} \mathrm{NiCl}_{2} \cdot 6 \mathrm{H}_{2} \mathrm{O}$ n.d.

$7 \quad$ No light n.d.

$8 \quad 440 \mathrm{~nm}, 24 \mathrm{~h} \quad 95$

$9 \quad 666 \mathrm{~nm}, 168 \mathrm{~h} \quad 95(92)^{a}$

B Characterization of DSMPs

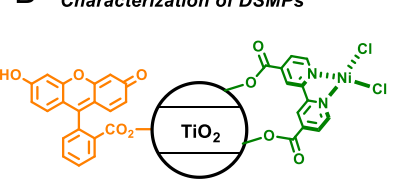

Fluo-DSMP

Dye loading: $\sim 64 \mu \mathrm{mol} \mathrm{g}^{-1}$

Nickel content: $\sim 131 \mu \mathrm{mol} \mathrm{g}$

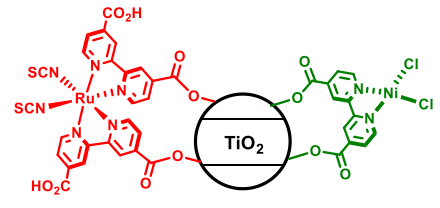

N3-DSMP

Dye loading: $\sim 66 \mu \mathrm{mol} \mathrm{g}^{-1}$ Nickel content: $\sim 199 \mu \mathrm{mol} \mathrm{g}^{-1}$
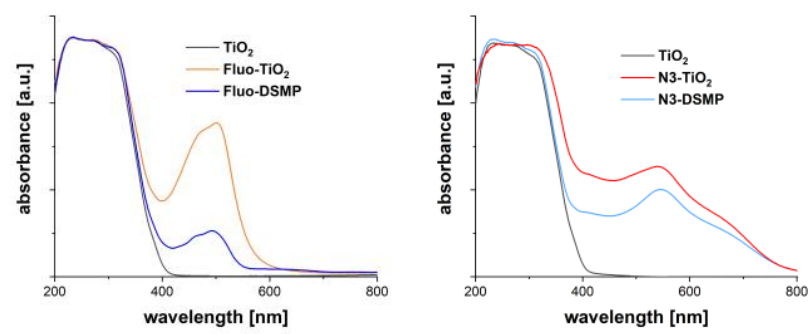

D C-S, C-N, and C-C cross couplings

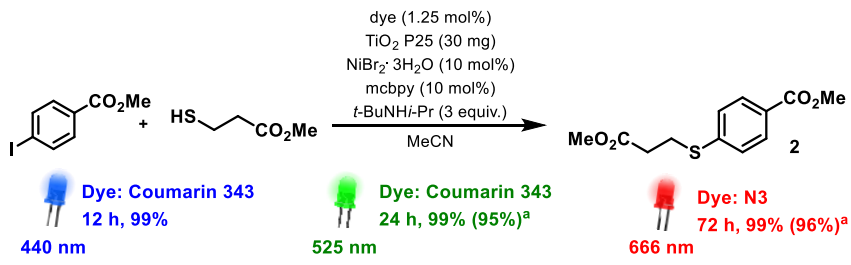

$440 \mathrm{~nm}$

$525 \mathrm{~nm}$

$666 \mathrm{~nm}$
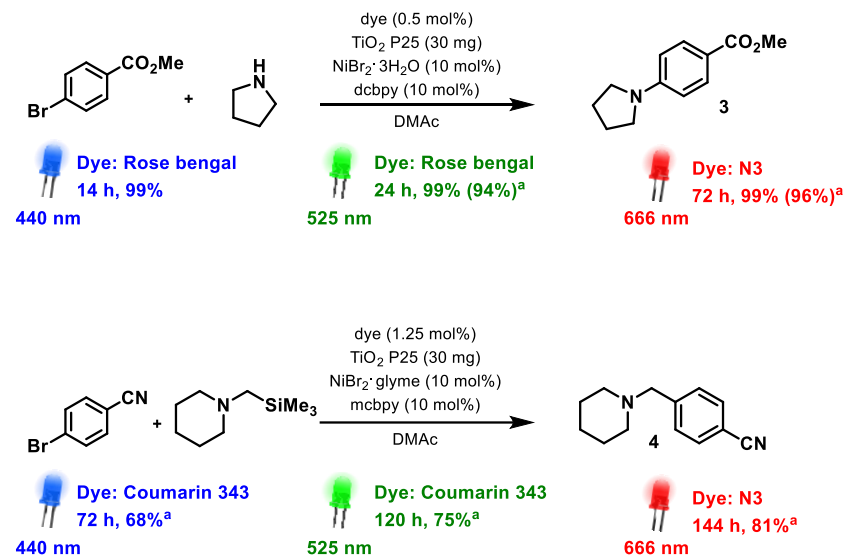

Fig. 2. Self-assembling, modular metallaphotocatalysts enable cross-couplings using the entire visible-light spectrum. Anchoring of nickel complexes on $\mathrm{TiO}_{2} \mathrm{P} 25$ improves yield due to permanent spatial proximity (A). UV-Vis spectra and nickel/dye loadings of two representative DSMPs (B). Optimized conditions and control experiments for the $O$-arylation of carboxylic acids using DSMPs. (D) $\mathrm{C}-\mathrm{S}, \mathrm{C}-\mathrm{N}$, and $\mathrm{C}-\mathrm{C}$ cross coupling catalyzed by DSMPs using blue, green, or red light. Yields were determined by ${ }^{1} \mathrm{H}-\mathrm{NMR}$ using 1,3,5trimethoxybenzene as internal standard if not stated otherwise. ${ }^{a}$ Isolated yields. DSMP $=$ dyesensitized metallaphotocatalyst n.d. $=$ not detected. dcbpy $=2,2^{\prime}$-bipyridine-4,4'-dicarboxylic acid. mcbpy = 4'-methyl-2,2'-bipyridine-4-carboxylic acid. glyme = 1,2-dimethoxyethane. $\mathrm{NaFluo}=$ fluorescein sodium 
The dynamic equilibrium between immobilized and unbound molecules is responsible for leaching of nickel and fluorescein during catalyst recycling studies and caused a gradual decrease of the yield of $\mathbf{1}$ (Fig. 3A). Addition of either NaFluo or the nickel salt restored the catalytic activity. This indicated that the amount of immobilized dye molecules and nickel complexes have to be above a certain limit to observe catalytic activity. We therefore questioned if only the close proximity of the dye molecules and the nickel complex is responsible for productive catalysis, and $\mathrm{TiO}_{2} \mathrm{P} 25$ only acts as support. In other words, an "onparticle" rather than a "through-particle" mechanism could be responsible for overcoming the short excited state lifetime (Fig. 3B). To test this hypothesis, we substituted $\mathrm{TiO}_{2} \mathrm{P} 25$ with the insulating metal oxides (MO) $\mathrm{SiO}_{2}$ and $\mathrm{Al}_{2} \mathrm{O}_{3}$ that only enable binding of the dye and the nickel complex and observed product formation for all cross-couplings, but with significantly lower efficiency compared to $\mathrm{TiO}_{2} \mathrm{P} 25$ (Fig. 3C). A second set of experiments was carried out using a nickel complex that is not able to bind to the surface of metal oxides. Here, only experiments with the semiconductor $\mathrm{TiO}_{2} \mathrm{P} 25$ gave productive catalysis. This confirms that dye sensitization leads to a charge-separated species that is sufficiently long-lived to turn over a homogeneous nickel catalyst in a semi-heterogeneous, diffusion-controlled reaction. Spectrophotometric titrations unveiled the electronic communication between the excited dye and the immobilized nickel complex "through" a semiconductor. Static fluorescence quenching of fluoresceinsensitized $\mathrm{TiO}_{2} \mathrm{P} 25$ was observed with a nickel complex that binds to the semiconductor's surface $\left(\mathrm{NiCl}_{2} \cdot \mathrm{dcbpy}\right) . \mathrm{NiCl}_{2} \cdot \mathrm{dtbbpy}$ showed solely dynamic quenching (Fig. 3D). Titration experiments with fluorescein bound to $\mathrm{SiO}_{2}$ instead of $\mathrm{TiO}_{2} \mathrm{P} 25$ displayed dynamic quenching behavior in case of both nickel complexes, and significantly lower quenching rates. Taking all results together, we assumed that a combination of "on-particle" and "through-particle" processes is responsible for the high catalytic activity of DSMPs with $\mathrm{TiO}_{2} \mathrm{P} 25$. 
A

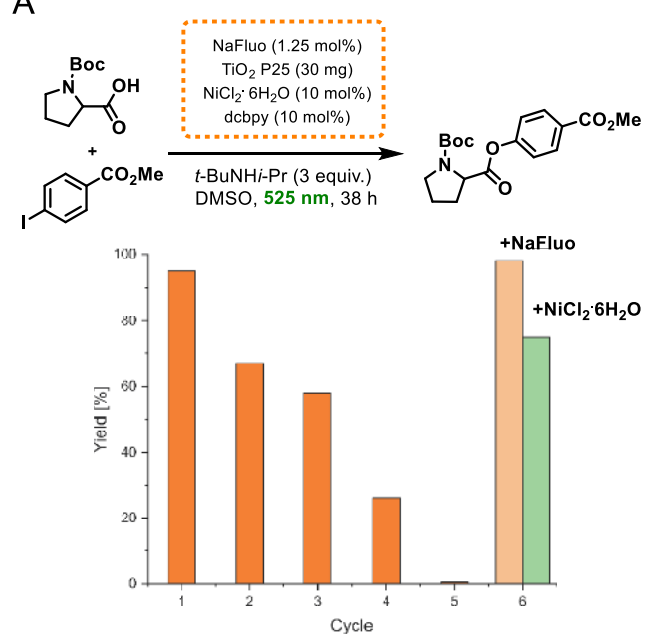

B

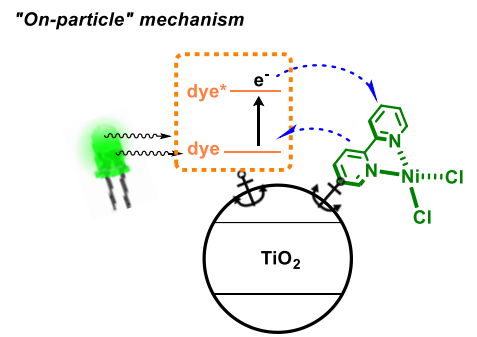

"Through-particle" mechanism

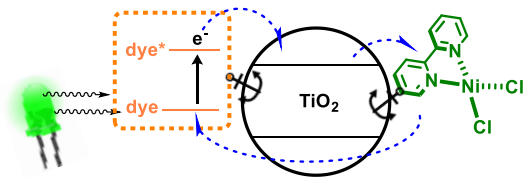

C
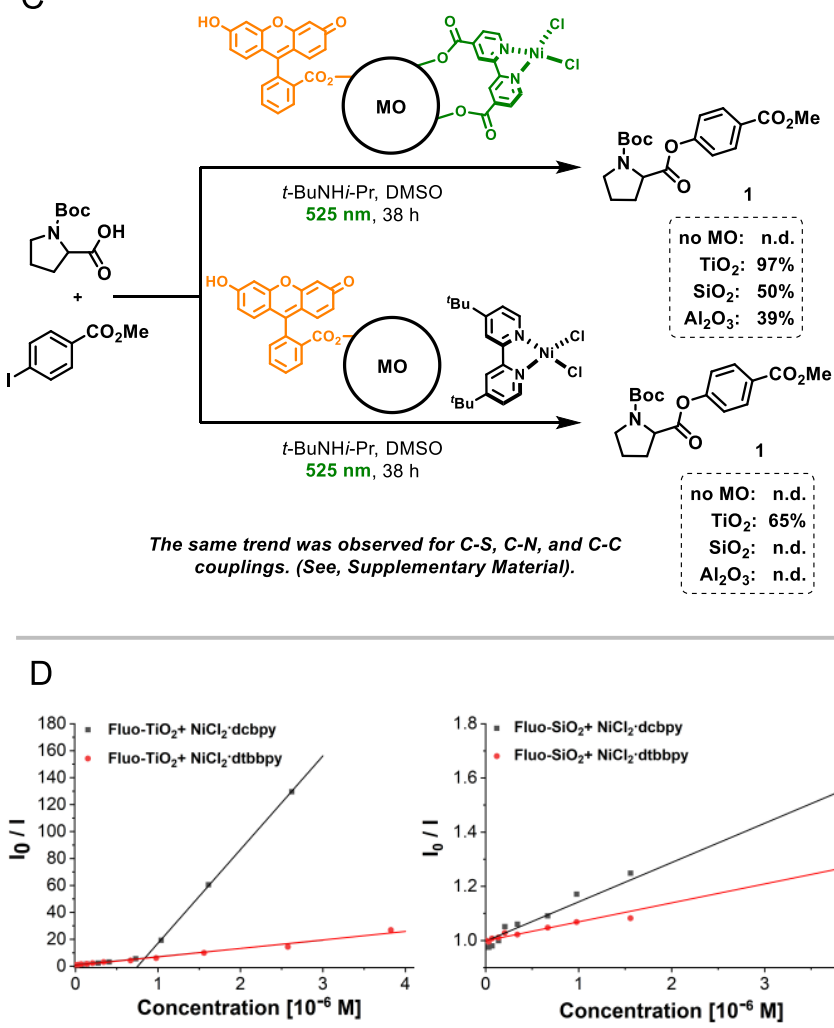

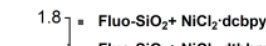
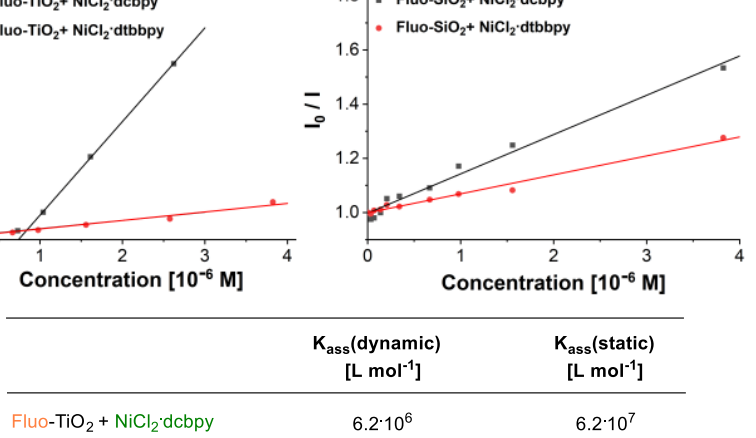

Fluo-

$6.2 \cdot 10^{6}$

Fluo- $\mathrm{SiO}_{2}+\mathrm{NiCl}_{2} \cdot \mathrm{dcbpy}$

$1.5 \cdot 10^{5}$

Fluo $-\mathrm{SiO}_{2}+\mathrm{NiCl}_{2} \cdot \mathrm{dtbbpy}$

Fig. 3. Mechanistic investigations. (A) Recycling experiments showed that catalytic inactive materials still contain nickel complexes and dye molecules. (B) DSMPs might work via an "onparticle" and/or "through-particle" mechanism. (C) Experiments using insulating metal oxides and diffusion controlled metallaphotocatalysis. (D) Spectrophotometric titrations show static quenching for DSMPs, whereas solely dynamic quenching was observed in other systems. MO $=$ metal oxide. dcbpy = 2,2'-bipyridine-4,4'-dicarboxylic acid. dtbbpy =4,4'-di-tert-butyl-2,2'bipyridine. Fluo = fluorescein .

In addition, we sought to compare the selectivity of DSMPs with homogenous metallaphotocatalysis systems in the $\mathrm{C}-\mathrm{O}$ arylation of $(E)$-cinnamic acid with 4iodobenzotrifluoride (Figure 4). The resulting coupling product $(\boldsymbol{E}-5)$ is prone to subsequent photocatalytic isomerization or cycloadditions that could lead to low selectivities $(18,19)$. We indeed observed significant amounts of the undesired Z-isomer (Z-5) when $\operatorname{Ir}\left(\mathrm{ppy}_{3}\right)$ was used as PCs using $440 \mathrm{~nm}$ irradiation. Other Ir complexes gave even worse results (see Supplementary Information). Control experiments showed that $\boldsymbol{E}-\mathbf{5}$ is also slowly converted to Z-5 at $440 \mathrm{~nm}$ in the absence of a PC (see Supplementary Information). The selectivity was, 
however, not improved using $\operatorname{Ir}\left(\mathrm{ppy}_{3}\right)$ and $525 \mathrm{~nm}$ irradiation, because the triplet energy of the PC is not wavelength-dependent. The $E-Z$ isomerization was also observed when the reaction was catalyzed by a DSMP at $440 \mathrm{~nm}$, but was totally suppressed by switching to higher wavelengths, resulting in a selective formation of $\boldsymbol{E}-\mathbf{5}$.

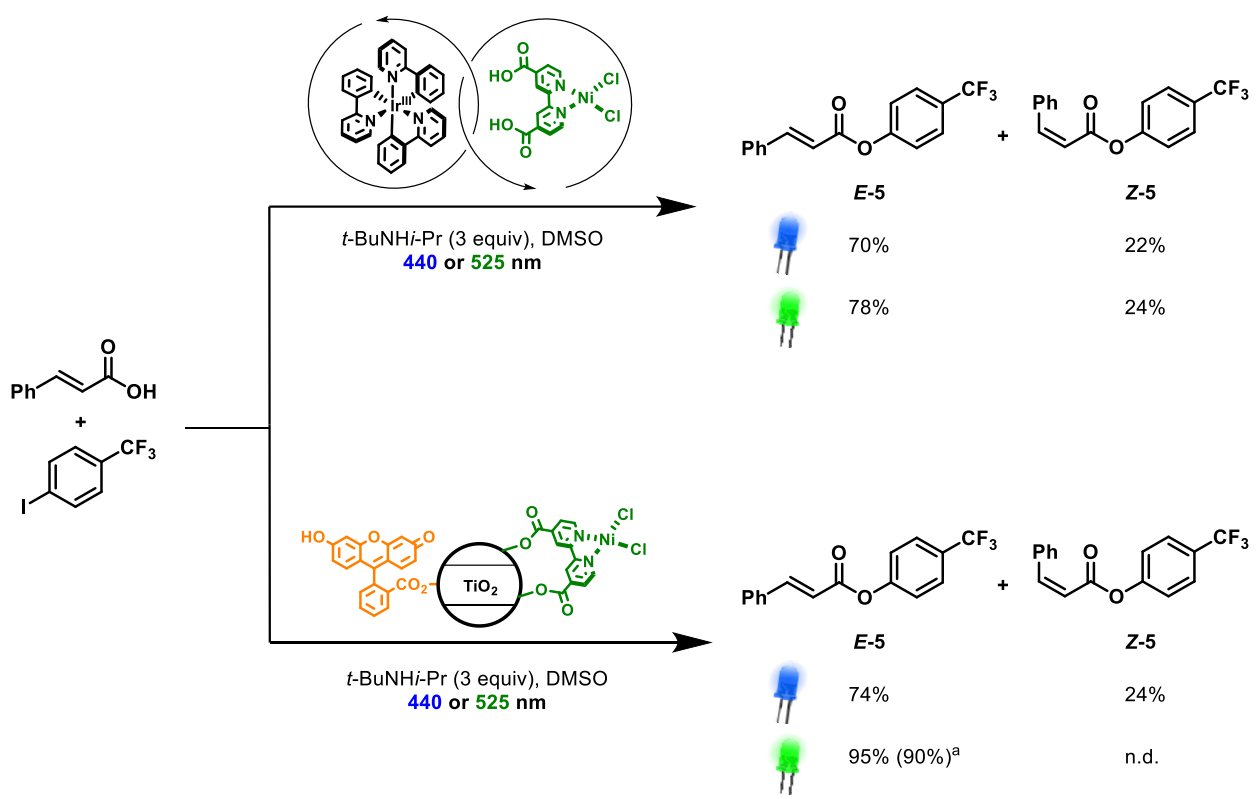

Figure 4. $\mathrm{C}-\mathrm{O}$ arylation of $(E)$-cinnamic acid with 4-iodobenzotrifluoride with different metallaphotocatalyst systems. Yields were determined by ${ }^{1} \mathrm{H}-\mathrm{NMR}$ using 1,3,5-

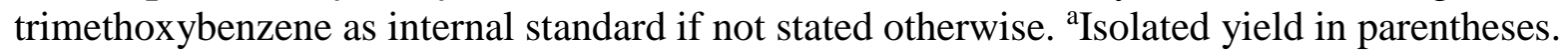

The DSMP concept overcomes the constraint of long excited state lifetimes of PCs for metallaphotocatalysis and unlocks the potential of many dyes that were previously unsuitable. Many cross couplings can be carried out using the entire visible light spectrum and selectivity issues can be tackled strategically. The simplicity and modularity of DSMPs suggest that the present approach will complement existing methods.

Acknowledgments: We gratefully acknowledge the Max-Planck Society for generous financial support. S.R. and B.P. thank the German Chemical Industry Fund (Fonds der Chemischen Industrie, FCI) for funding through a Liebig Fellowship. B.P. thanks the Deutsche Forschungsgemeinschaft (DFG, German Research Foundation) under Germany's Excellence Strategy - EXC 2008 - 390540038 - UniSysCat for financial support. We thank our colleagues Prof. Peter H. Seeberger, Dr. Jamal Malik, Dr. Martina Delbianco, Dr. Kerry Gilmore, Dr. Lukas Zeininger, Cristian Cavedon, Silvia Fürstenberg, Jessica Brandt and Katharina ten Brummelhuis (all MPIKG), for scientific, technical and analytical support. 


\section{References:}

1. J. A. Milligan, J. P. Phelan, S. O. Badir, G. A. Molander, Alkyl Carbon-Carbon Bond Formation by Nickel/Photoredox Cross-Coupling. Angew. Chem. Int. Ed. 58, 61526163 (2019).

2. J. Twilton et al., The merger of transition metal and photocatalysis. Nat. Rev. Chem. 1, 1-18 (2017).

3. C. Cavedon, P. H. Seeberger, B. Pieber, Photochemical Strategies for CarbonHeteroatom Bond Formation. Eur. J. Org. Chem. 10, 1379-1392 (2020).

4. J. Lu et al., Donor-Acceptor Fluorophores for Energy-Transfer-Mediated Photocatalysis. J. Am. Chem. Soc. 140, 13719-13725 (2018).

5. F. Glaser, C. Kerzig, O. S. Wenger, Multi-Photon Excitation in Photoredox Catalysis: Concepts, Applications, Methods. Angew. Chem. Int. Ed. 59, 2-21 (2020).

6. B. D. Ravetz et al., Photoredox catalysis using infrared light via triplet fusion upconversion. Nature. 565, 343-346 (2019).

7. B. D. Ravetz et al., https://doi.org/10.26434/chemrxiv.12124215.v1. (2020).

8. N. A. Romero, D. A. Nicewicz, Organic Photoredox Catalysis. Chem. Rev. 116, 10075-10166 (2016).

9. A. Hagfeldt, G. Boschloo, L. Sun, L. Kloo, H. Pettersson, Dye-Sensitized Solar Cells. Chem. Rev. 110, 6595-6663 (2010).

10. J. Willkomm et al., Dye-sensitised semiconductors modified with molecular catalysts for light-driven $\mathrm{H} 2$ production. Chem. Soc. Rev. 45, 9-23 (2016).

11. A. Hagfeldt, M. Graetzel, Light-Induced Redox Reactions in Nanocrystalline Systems. Chem. Rev. 95, 49-68 (1995).

12. E. R. Welin, C. Le, D. M. Arias-Rotondo, J. K. McCusker, D. W. C. MacMillan, Photosensitized, energy transfer-mediated organometallic catalysis through electronically excited nickel(II). Science 355, 380-385 (2017).

13. X. Zhu et al., Lead halide perovskites for photocatalytic organic synthesis. Nat. Commun. 10, 2843 (2019).

14. B. Pieber et al., Semi-heterogeneous Dual Nickel/Photocatalysis using Carbon Nitrides: Esterification of Carboxylic Acids with Aryl Halides. Angew. Chem. Int. Ed. 58, 9575-9580 (2019).

15. M. S. Oderinde, M. Frenette, D. W. Robbins, B. Aquila, J. W. Johannes, Photoredox Mediated Nickel Catalyzed Cross-Coupling of Thiols With Aryl and Heteroaryl Iodides via Thiyl Radicals. J. Am. Chem. Soc. 138, 1760-1763 (2016).

16. E. B. Corcoran et al., Aryl amination using ligand-free Ni(II) salts and photoredox catalysis. Science 353, 279-283 (2016).

17. C. Remeur, C. B. Kelly, N. R. Patel, G. A. Molander, Aminomethylation of Aryl Halides Using $\alpha$-Silylamines Enabled by Ni/Photoredox Dual Catalysis. ACS Catalysis 7, 6065-6069 (2017).

18. T. Lei et al., General and Efficient Intermolecular [2+2] Photodimerization of Chalcones and Cinnamic Acid Derivatives in Solution through Visible-Light Catalysis. Angew. Chem. Int. Ed. 56, 15407-15410 (2017).

19. P. Shu et al., Synthesis of (Z)-Cinnamate Derivatives via Visible-Light-Driven E-to-Z Isomerization. SynOpen 03, 103-107 (2019). 